\title{
CARACTERIZACIÓN DE LA DINÁMICA DE FLUJO MEDIANTE LA APLICACIÓN DE UN MODELO NUMÉRICO HIDROGEOLÓGICO. CASO DE LA CUENCA DEL RÍO BIRRÍS, CARTAGO, COSTA RICA
}

\author{
Pablo Ramírez Granados \\ Escuela de Geografía - Observatorio del Desarrollo \\ Apdo. 214-2060-Universidad de Costa Rica \\ Correo-e: geohidromath@gmail.com
}

(Recibido 20/04/07; aceptado 02/05/07)

\begin{abstract}
The numerical model application in Microsoft Excel $($ is analyzed as an alternative to understand the groundwater flow dynamics in the Birrís river watershed. Because of lack of hydrogeological studies, a conceptual model was defined starting from geological and hydrogeological units, springs lithology and emergence and its fluoride concentration. In order to construct a model, springs and drainage elevations of the zone were used and finite differences were applied to solve the Laplace equation. The model results show several effluent hydraulic connection zones in different places of the watershed, which also were related with the outflow increase. The model was compared with a statistical model to show how the first one exhibits a lot of advantages compared to the second one, when it is utilized in groundwater modeling.

Key words: Flow numerical model, Laplace equation, finite differences, Birrís river watershed, aquifer system, Birrís, Pacayas-Reventado.

RESUMEN: Se analiza la aplicación de un modelo numérico desarrollado en Microsoft Excel@ como una alternativa para conocer la dinámica de flujo de las aguas subterráneas en la cuenca del río Birrís. Debido a la carencia de estudios hidrogeológicos, se definió un modelo conceptual a partir de la información sobre las unidades geológicas e hidrogeológicas, la litología y el afloramiento de manantiales y las concentraciones de fluoruro en ellos. Para la construcción del modelo se utilizaron niveles de manantiales y drenajes superficiales de la zona y se aplicaron diferencias finitas para resolver la ecuación de Laplace. Los resultados del modelo muestran varias zonas de conexión hidráulica efluente en distintos puntos de la cuenca, lo cual también se relaciona con el aumento de caudal. El modelo numérico fue comparado con un modelo estadístico para demostrar cómo el primero presenta mayores ventajas sobre el segundo cuando se realiza un modelado hidrogeológico.

Palabras clave: Modelo numérico de flujo, ecuación de Laplace, diferencias finitas, cuenca río Birrís, sistema acuífero, Birrís, Pacayas-Reventado.
\end{abstract}




\section{INTRODUCCIÓN}

En la construcción de un modelo hidrogeológico conceptual, el conocimiento sobre las equipotenciales y la dirección de flujo de las aguas subterráneas es muy importante para determinar la dinámica del flujo hidrogeológico (Bear, 1979). Esta información ayuda a conocer las posibles zonas de descarga, las relaciones hidráulicas entre cuerpos de agua como ríos o lagunas, el tiempo de tránsito de contaminantes y la zona de captura de un pozo, entre otros.

Generalmente, para la construcción de las redes de flujo se ha partido del conocimiento del valor del nivel estático en un pozo. De esta manera, utilizando un conjunto de estos valores, se puede generar un mapa de equipotenciales.

Sin embargo, en regiones donde no existen pozos o estos son muy pocos, es necesario recurrir a otros métodos como la distribución de los manantiales (Bear, 1979; Anderson \& Woessner, 1992), los cuales brindan mucha información sobre los procesos geológicos e hidrológicos (Manga, 2001). Una manera para determinar la dinámica de flujo en estas regiones consiste en utilizar la ecuación de Laplace (Bear, 1979). Esta ecuación describe el flujo de un fluido incompresible en una matriz homogénea e isotrópica (Bear, 1972, 1979).

\section{Construcción de un modelo numérico de flujo}

La aplicación de un modelo numérico para la caracterización de la dinámica de flujo de las aguas subterráneas está basada en la resolución de la ecuación de Laplace que gobierna el flujo en régimen permanente en un acuífero (Polubarinova-Kochina, 1962; Bear, 1972, 1979). Para la confección del modelo es necesario resolver esta ecuación utilizando diversas técnicas de análisis numérico, basadas principalmente en los métodos de resolución por diferencias finitas y elementos finitos (Bear, 1979), aunque existen algunos otros, como diferencias finitas integradas (Marsily, 1986) y el método de la ecuación integral de frontera (Anderson \& Woessner, 1992). En este artículo se enfocará la utilización del método de las diferencias finitas para caracterizar el flujo de aguas subterráneas en régimen permanente.
El método de diferencias finitas o promedios aritméticos (Polubarinova-Kochina, 1962) es una herramienta de fácil entendimiento y programación, permite comprender el flujo de agua subterránea regional en una o dos dimensiones, o en sistemas multicapas en tres dimensiones. El método puede ser utilizado para resolver el problema de flujo en régimen permanente o en régimen transitorio (Bear, 1979; Marsily, 1986; Anderson \& Woessner, 1992).

El método para flujo permanente consiste en considerar la ecuación diferencial parcial para régimen de flujo permanente o ecuación diferencial de Laplace:

$$
d^{2} h / d x^{2}+d^{2} h / d y^{2}+d^{2} h / d z^{2}=0
$$

El significado físico de esta ecuación es que el acuífero está en una condición de equilibrio y estabilidad, de forma que el cambio de almacenamiento no varía y que la carga hidráulica no cambia con el tiempo. Se considera el acuífero isotrópico, de composición uniforme y con conductividad hidráulica constante (Bear, 1972, 1979; Knapp, 1999). Para la resolución de esta ecuación es importante considerar un dominio limitado o finito. Una vez establecido el dominio finito, se realiza una discretización del espacio (fig. 1) y se resuelve la ecuación diferencial parcial mediante el método de las diferencias finitas (Polubarinova-Kochina, 1962; Bear, 1972, 1979; Marsily, 1986, Anderson \& Woessner, 1992; Knapp, 1999).

Para una celda individual del dominio finito discretizado (Fig. 1), considerando en ella un flujo bidimensional esencialmente horizontal, un área unitaria y una dirección de flujo de $\mathrm{H}_{1}$ a $\mathrm{H}_{3}$ en el eje $\mathrm{X}$ y de $\mathrm{H}_{4}$ a $\mathrm{H}_{2}$ en el eje $\mathrm{Y}$, sin considerar variaciones de elevación en la carga hidráulica $\left(\mathrm{d}^{2} \mathrm{~h} / \mathrm{dz}^{2}=0\right)$, se tiene entonces lo siguiente:

Para las celdas dentro del dominio discretizado, la solución es la siguiente (PolubarinovaKochina, 1962; Bear, 1972, 1979; Marsily, 1986; Knapp, 1999):

$$
\begin{aligned}
& \mathrm{d}^{2} \mathrm{~h} / \mathrm{dx}{ }^{2}=\left(\mathrm{H}_{1}-\mathrm{H}_{0}\right) / \mathrm{L}-\left(\mathrm{H}_{0}-\mathrm{H}_{3}\right) / \mathrm{L} \\
& \mathrm{d}^{2} \mathrm{~h} / \mathrm{dy}^{2}=\left(\mathrm{H}_{4}-\mathrm{H}_{0}\right) / \mathrm{L}-\left(\mathrm{H}_{0}-\mathrm{H}_{2}\right) / \mathrm{L}
\end{aligned}
$$




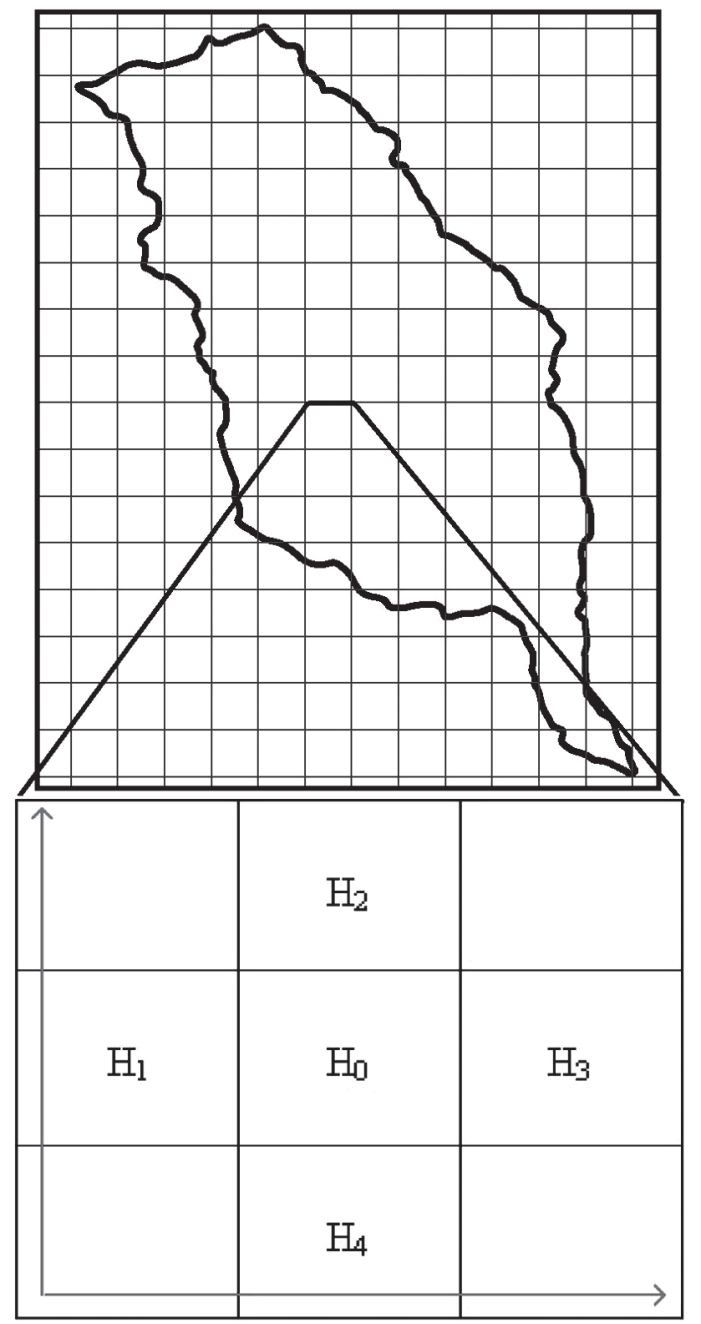

Fig. 1: Dominio finito discretizado y celda individual de flujo. Nótese en color negro el contorno del espacio finito, el cual ha sido discretizado y la forma en la cual se aplica el método de diferencias finitas por celda de área unitaria. Se indica la dirección bidimensional de flujo.

Combinando ambas ecuaciones y despejando la variable $\mathrm{H}_{0}$, la ecuación numérica para flujo en régimen permanente será la siguiente:

$$
\mathrm{H}_{0}=\left(\mathrm{H}_{1}+\mathrm{H}_{2}+\mathrm{H}_{3}+\mathrm{H}_{4}\right) / 4
$$

El significado de esta ecuación numérica es que la carga hidráulica en cada celda en la cual se aplicó la diferencia finita, es igual al promedio de las cargas en las cuatro celdas vecinas
(Polubarinova-Kochina, 1962; Anderson \& Woessner, 1992). Las condiciones de frontera son especificadas considerando el límite de la cuenca impermeable y los manantiales y drenajes superficiales como zonas de descarga de las aguas subterráneas (Bear, 1979; Marsily 1986; Anderson \& Woessner, 1992).

Actualmente existen muchos paquetes computacionales que permiten resolver la ecuación de flujo mediante diferencias finitas, sin embargo muchos de ellos consideran un conocimiento muy detallado del medio a modelar (Anderson \& Woessner, 1992) y presentan en algunos casos un costo económico muy significativo. Schosinsky (com. pers., 2006) desarrolló una metodología para construir un modelo hidrogeológico de flujo utilizando Microsoft ExcelC 0 . De esta forma, mediante una hoja electrónica de cálculo se insertan tanto las ecuaciones numéricas, como los parámetros hidrogeológicos del modelo conceptual del acuífero. Este modelo será utilizado para estudiar la dinámica de flujo en régimen permanente en la cuenca del río Birrís, Cartago, Costa Rica.

\section{Aplicación del modelo numérico de flujo en la cuenca del río Birrís}

La cuenca del río Birrís se ubica en la vertiente sureste del volcán Irazú, en la provincia de Cartago (Fig. 2), entre las coordenadas $551000-561000$ este y $205000-218000$ norte, según el sistema de proyección cartográfico cónica conforme de Lambert Costa Rica Norte. Debido a que en la cuenca del río Birrís no existen pozos registrados, se utilizó el modelo numérico de flujo en Microsoft Excel(C) como una alternativa para poder describir la dinámica de las aguas subterráneas en esta cuenca. El modelo usando Microsoft Excel(C) funciona con pocos datos y permite describir el flujo considerando únicamente régimen permanente, además se le pueden integrar a futuro otras variables como recarga, descarga y transmisividad, entre otros, y conforme se avance en el conocimiento del modelo hidrogeológico conceptual de la cuenca, se puede convertir a modelo transitorio. 


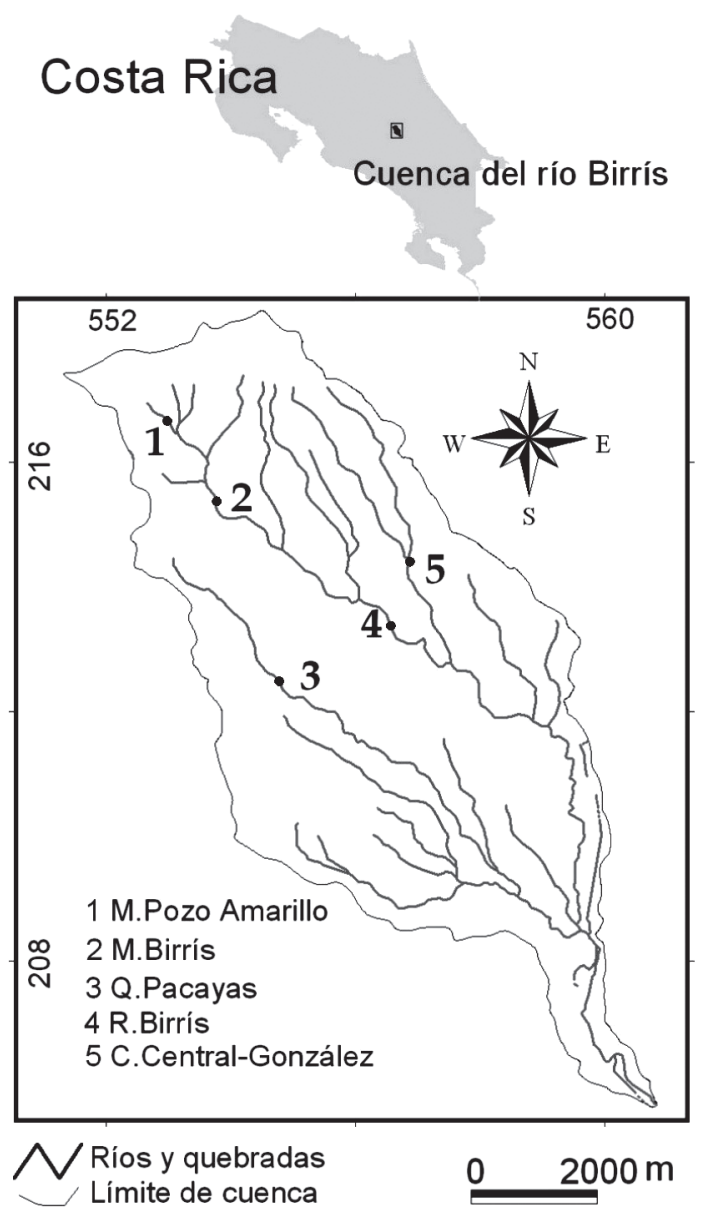

Fig. 2: Mapa de ubicación de la cuenca del río Birrís. Se observan las principales zonas de manantiales y los drenajes superficiales más importantes.

\section{ESTRATIGRAFÍA DE LA CUENCA DEL RÍO BIRRÍS}

Krushensky (1972) definió la estratigrafía para la hoja Istarú (escala 1: 50000 del Instituto Geográfico Nacional, IGN) dentro de la cual se encuentra la cuenca del río Birrís. En esta cuenca se encuentran las formaciones Ujarraz, Reventado (Miembros Paraíso, Capa de Ceniza y Superior), Sapper, Birrís y Cervantes. El Miembro Superior de Reventado, Sapper, Birrís y Cervantes constituyen más del 95\% del área total (Fig. 3). Debido a esta característica se describirán estas cuatro formaciones.

\section{Formación Reventado, Miembro Superior}

Conformada por flujos de lava y capas intermedias de lahares, así como depósitos de ceniza. Las lavas son de color gris oscuro a gris claro y son definidas como lavas andesítico basálticas, con un tamaño de cristales que varía de grueso a fino. Presentan espesores que varían desde 5 a $15 \mathrm{~m}$, en algunos tramos de los ríos y quebradas, hasta más de $50 \mathrm{~m}$ en las cataratas y los sectores encañonados de los ríos. Los lahares muestran de 1 a $2 \mathrm{~m}$ de espesor aflorando en los cañones de los ríos y presentan matriz y bloques suaves y fácilmente erosionables (Krushensky, 1972). Además se reconocen fragmentos de roca que varían desde partículas milimétricas hasta bloques métricos, que pueden ser desde roca dura y angular hasta bloques redondeados y subredondeados,

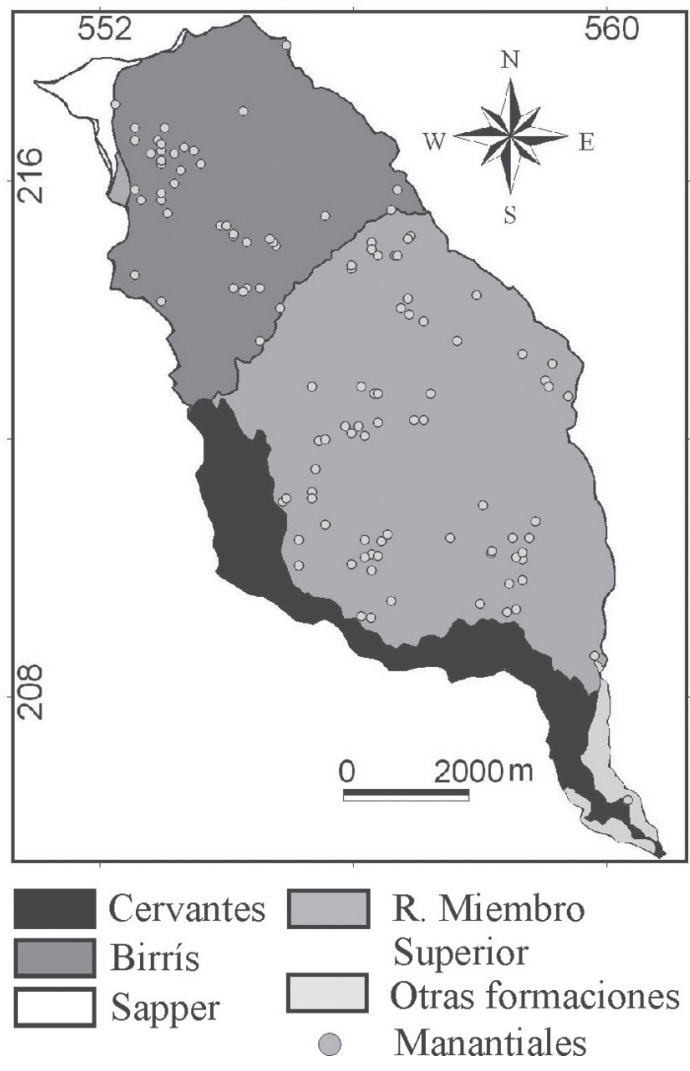

Fig. 3: Mapa geológico modificado de Krushensky (1972). Nótense las unidades geológicas y los manantiales. 
que se muestran muy meteorizados. Comúnmente contienen lentes de arcilla, de color gris a gris verdosa, que presentan propiedades de alta plasticidad cuando están humedecidas (Krushensky, 1972). Las capas de ceniza afloran en toda la superficie, principalmente en los cortes de los caminos y muestran espesores variables de hasta 3 m. Estas cenizas son finas (Clark, 1993), de color oscuro y su textura es arenosa, con cierto contenido de materia orgánica. Estudios sugieren que la edad del Miembro Superior está entre 143-147 ka (Krushensky, 1972) ó 110 ka, según análisis radiométricos de ${ }^{238} \mathrm{U} /{ }^{230} \mathrm{Th}$ (Allègre \& Condomines, 1976).

\section{Formación Sapper}

Está conformada por flujos de lavas con capas intermedias de lahares y cenizas, que sobreyacen disconformemente a la Formación Reventado y se encuentran sobreyacidos disconformemente por la Formación Birrís. (Krushensky, 1972). Las lavas son densas, de color negro verdoso oscuro a negro y café rojizo, cuando se encuentran meteorizadas. Los lahares dentro de esta formación contienen clastos de las lavas de la Formación Reventado: fragmentos líticos que varían desde arenas a bloques métricos y con distintos grados de meteorización (Krushensky, 1972). Según Alvarado (1993), la edad probable de esta formación está en el rango de $68 \pm 26 \mathrm{ka}$ y $36 \pm 14 \mathrm{ka}$.

\section{Formación Birrís}

Son lavas andesíticas afaníticas porfiríticas, que sobreyacen disconformemente a la Formación Sapper y están sobreyacidas conformemente por la Formación Cervantes (Krushensky, 1972). Esta formación consiste de flujos de lavas de color gris oscuro a gris medio. Los espesores de estas lavas varían desde $5 \mathrm{~m}$ en la naciente del río Birrís, hasta más de $50 \mathrm{~m}$ en las cataratas y cañones que hay en el trayecto del río. Los afloramientos de esta formación están concentrados en los ríos y algunos cortes de caminos.

\section{Formación Cervantes}

Está conformada por flujos de lavas escoriáceas y blocosas superficialmente, de color oscuro que sobreyacen a las Formaciones Birrís y Reventado, aflorando desde el sector del cerro Pasquí hasta el cañón del río Reventazón. Esta formación se caracteriza por presentar un desarrollo de suelos mínimo y restringido a la meteorización de las cenizas posteriores a las lavas de Cervantes (Krushensky, 1972).

Son el complejo de lavas más recientes del Irazú, y se divide en dos unidades: la oeste es basáltica con una edad $\mathrm{Ar} / \mathrm{Ar}$ de $57 \pm 13 \mathrm{ka}$, y la este con una edad Ar/Ar de $20 \pm 12 \mathrm{ka}$, y edades calibradas de radiocarbono de 16840 años (Alvarado et al, 2006), similares a los 23000 años según dataciones de uranio-torio $\left({ }^{238} \mathrm{U} / 230 \mathrm{Th}\right)$ en las muestras de lava (Allègre \& Condomines, 1976; Alvarado, 1993).

\section{MODELO HIDROGEOLÓGICO CONCEPTUAL}

Para definir el modelo hidrogeológico conceptual de la cuenca del río Birrís, se definieron las unidades hidrogeológicas y los sistemas acuíferos que existen en la cuenca del río Birrís. En esta cuenca no existen estudios hidrogeológicos anteriores que sirvan de base para esta investigación.

\section{Unidades hidrogeológicas}

La definición de las unidades hidrogeológicas requiere el conocimiento de los estratos litológicos, su espesor y conductividad hidráulica. Esta información en algunos casos es muy difícil de obtener, como en el caso de la cuenca del río Birrís, por lo que para establecer estas unidades se puede recurrir a otros criterios hidrogeológicos (Davis \& De Wiest, 1966; Bear, 1979; Marsily, 1986; Anderson \& Woessner, 1992; Custodio \& Llamas, 2001; ASTM, 2004) así como geológicos (Thorpe \& Brown, 1985). 
Los criterios utilizados para determinar las unidades hidrogeológicas de la cuenca del río Birrís fueron: afloramiento y caudal de manantiales, tipo de litología, propiedades físicas macroscópicas de la roca, y relaciones espacio-temporales de las unidades volcánicas, entre otros. Las unidades hidrogeológicas definidas son las siguientes:

\section{Lavas Pozo Amarillo-Birrís}

Se encuentran aflorando en toda la Formación Birrís. Son lavas andesíticas fracturadas y brechosas que muestran grados de saturación variables, los caudales de los manantiales principales varían entre 15 y 40 L/s (MINAE, 2006) y logran abastecer a varias poblaciones de la zona. Además existen otros manantiales que presentan caudales menores de $0,5 \mathrm{~L} / \mathrm{s}$.

Los afloramientos de estas lavas están expuestos tanto por tajos artesanales como por los cañones, principalmente en el cañón del río Birrís (Fig. 4) y en algunas quebradas aledañas como Laguna Tapada, Central y González. El espesor de esta unidad es variable, en los manantiales de Pozo Amarillo, las lavas alcanzan un espesor aproximado de $15 \mathrm{~m}$, en tanto en los manantiales Birrís, en la catarata, el espesor puede alcanzar más de $60 \mathrm{~m}$
(Fig. 4). La mayoría de los manantiales observados afloran tanto en el contacto entre lavas fracturadas y brechas, como directamente en lavas fracturadas, como en el caso de los manantiales de Pozo Amarillo y Birrís. La permeabilidad de esta unidad está favorecida por el fracturamiento causado por el enfriamiento de las corrientes de lava parcialmente solidificadas (Davis \& De Wiest, 1966), así como por la textura gruesa de las brechas, la cual presenta fragmentos de tamaños centimétricos hasta decimétricos con porosidad interpartícula.

Considerando los brotes de manantiales a lo largo del río Birrís y los depósitos de lavas fracturadas y brechosas de espesores métricos variables, se puede decir que estas lavas actúan como un acuífero.

\section{Lavas Pacayas-Reventado}

Estas lavas son parte del Miembro Superior de la Formación Reventado, macroscópicamente son lavas andesítico basálticas fracturadas y brechosas que se asemejan a las lavas Pozo Amarillo-Birrís. En el río Birrís los manantiales corresponden a extensas zonas de manantiales con caudales menores a $0,5 \mathrm{~L} / \mathrm{s}$. El espesor de esta unidad es muy variado, en

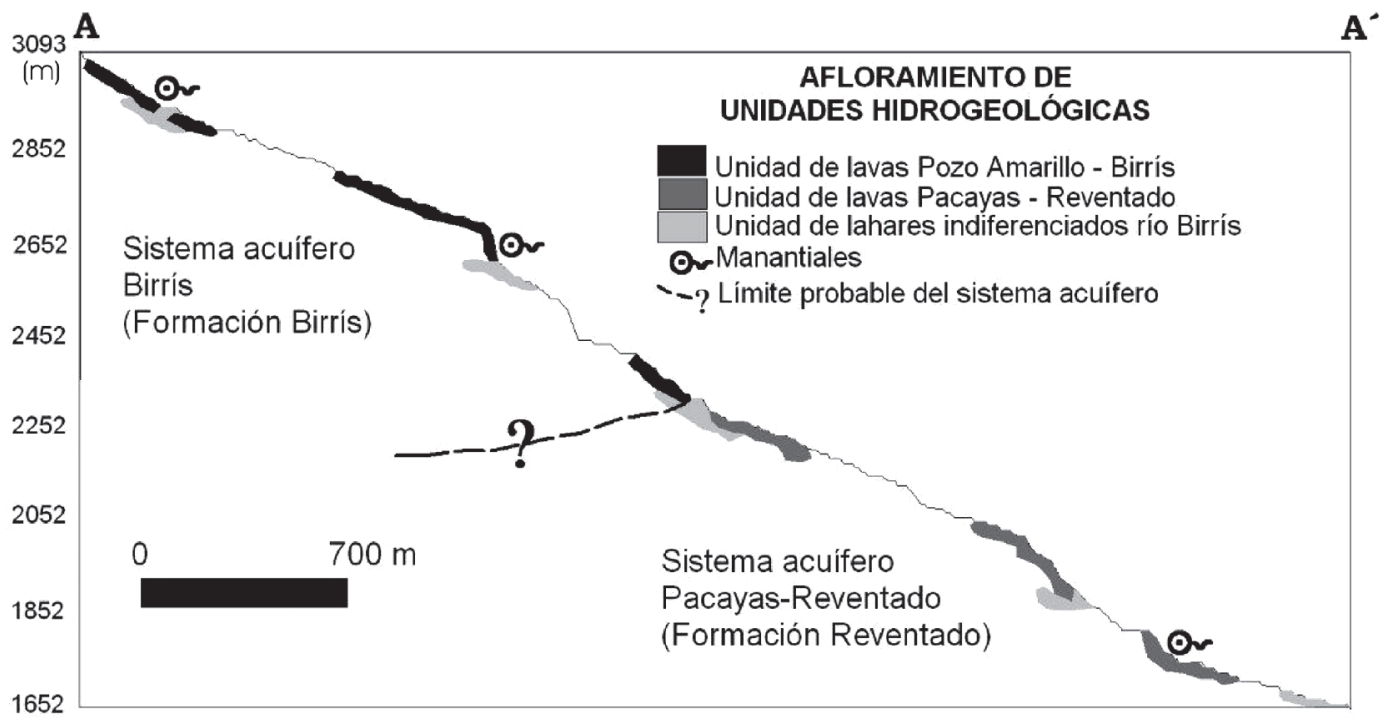

Fig. 4: Sección con unidades hidrogeológicas A-A' del río Birrís, mostrado en planta en la figura 7, en el que se muestran las unidades hidrogeológicas y los sistemas acuíferos. 
diferentes tramos a lo largo de los ríos varía entre 10 y $20 \mathrm{~m}$, mientras en los sectores de catarata y fuertemente encañonados llega a alcanzar más de $70 \mathrm{~m}$. Los principales afloramientos de agua de estas lavas se encuentran en la confluencia Central-González (Fig. 5) con caudales que varían entre 22 y $80 \mathrm{~L} / \mathrm{s}$ (MINAE, 2006) y en el curso medio de la quebrada Pacayas y el río Ortiga (Fig. 6) con caudales promedio de 22 L/s (MINAE, 2006). Los manantiales en esta unidad se originan del contacto entre lavas brechosas y fracturadas o en lavas fracturadas. Además fueron observados zonas de manantiales, con caudales menores de $0,5 \mathrm{~L} / \mathrm{s}$, en el contacto entre esta unidad y los lahares indiferenciados del río Birrís.

$\mathrm{Al}$ igual que la unidad de Lavas Pozo AmarilloBirrís, las lavas Pacayas-Reventado muestran alta permeabilidad debido al fracturamiento de los materiales lávicos y a la textura gruesa de las brechas. Considerando las características de esta unidad, se puede concluir que se comporta como un acuífero.

\section{Lavas meteorizadas Pacayas - Ortiga}

Son parte de la Formación Reventado, Miembro Superior y consiste de depósitos de lavas muy meteorizados, los cuales macroscópicamente muestran una textura muy plástica y cohesiva, se diferencian de los lahares debido a que el depósito es más granular y se conservan los piroxenos y las plagioclasas, aunque en algunos sectores es fácilmente confundible con la unidad de lahares indiferenciados Pacayas-González. El espesor observado de estas lavas varía entre 2 y 6 $\mathrm{m}$. Esta unidad aflora cerca de la quebrada Lajas y en la quebrada que se encuentra al este del río Ortiga. Debido a su plasticidad y cohesividad, los materiales derivados de estas lavas meteorizadas actúan como un acuitardo.

\section{Cenizas Irazú}

Se encuentran en la Formación Birrís. Son cenizas recientes originadas por la actividad volcánica del Irazú. Conforman espesores métricos variables según la topografía y la pendiente. Aunque se encuentran en toda la superficie de la Formación Birrís, su representatividad está en función del uso de la tierra actual, ya que en la mayoría de la cuenca la remoción del suelo llega a alcanzar hasta $50 \mathrm{~cm}$. En el sector más alto de la

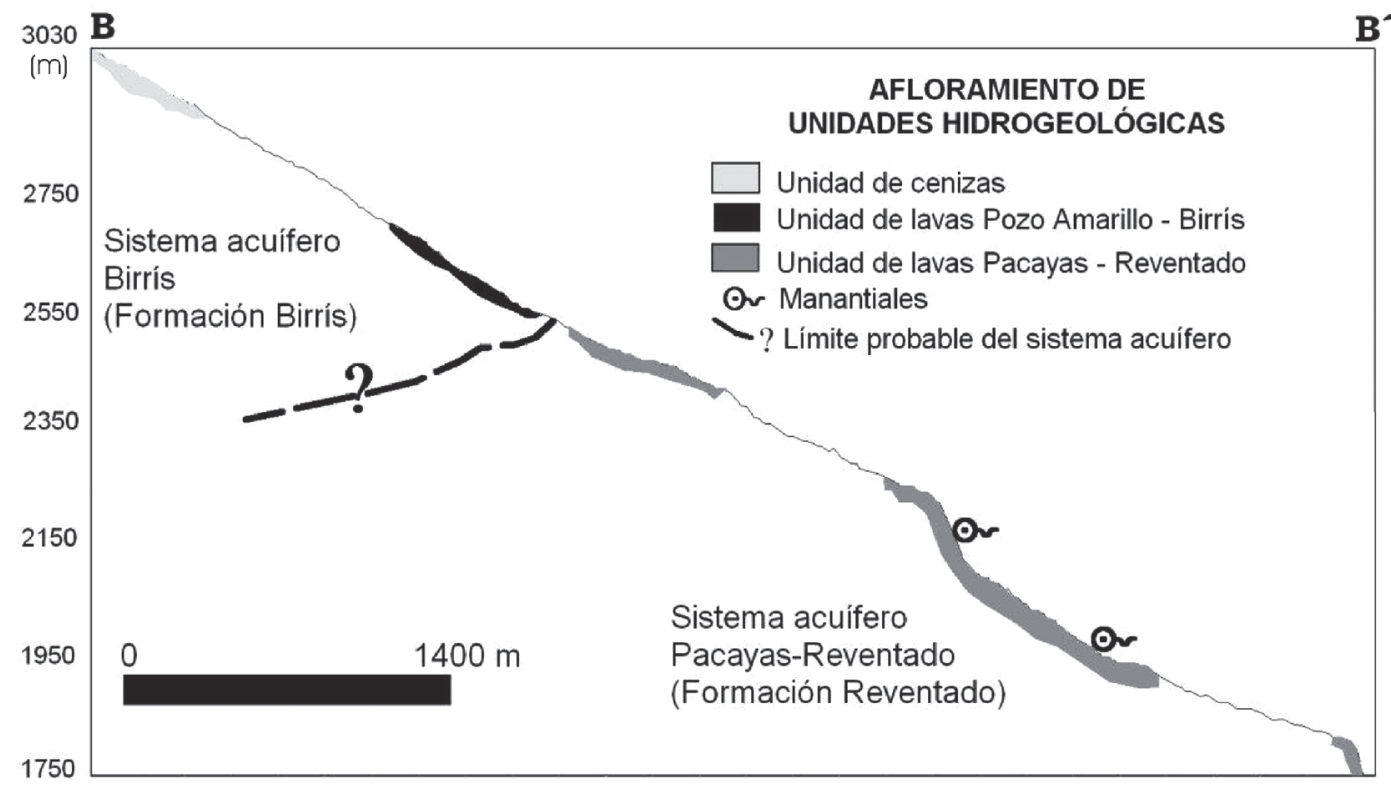

Fig. 5: Sección con unidades hidrogeológicas B-B’ de la confluencia Central-González, mostrado en planta en la figura 7, en el que se muestran las unidades hidrogeológicas y los sistemas acuíferos. 


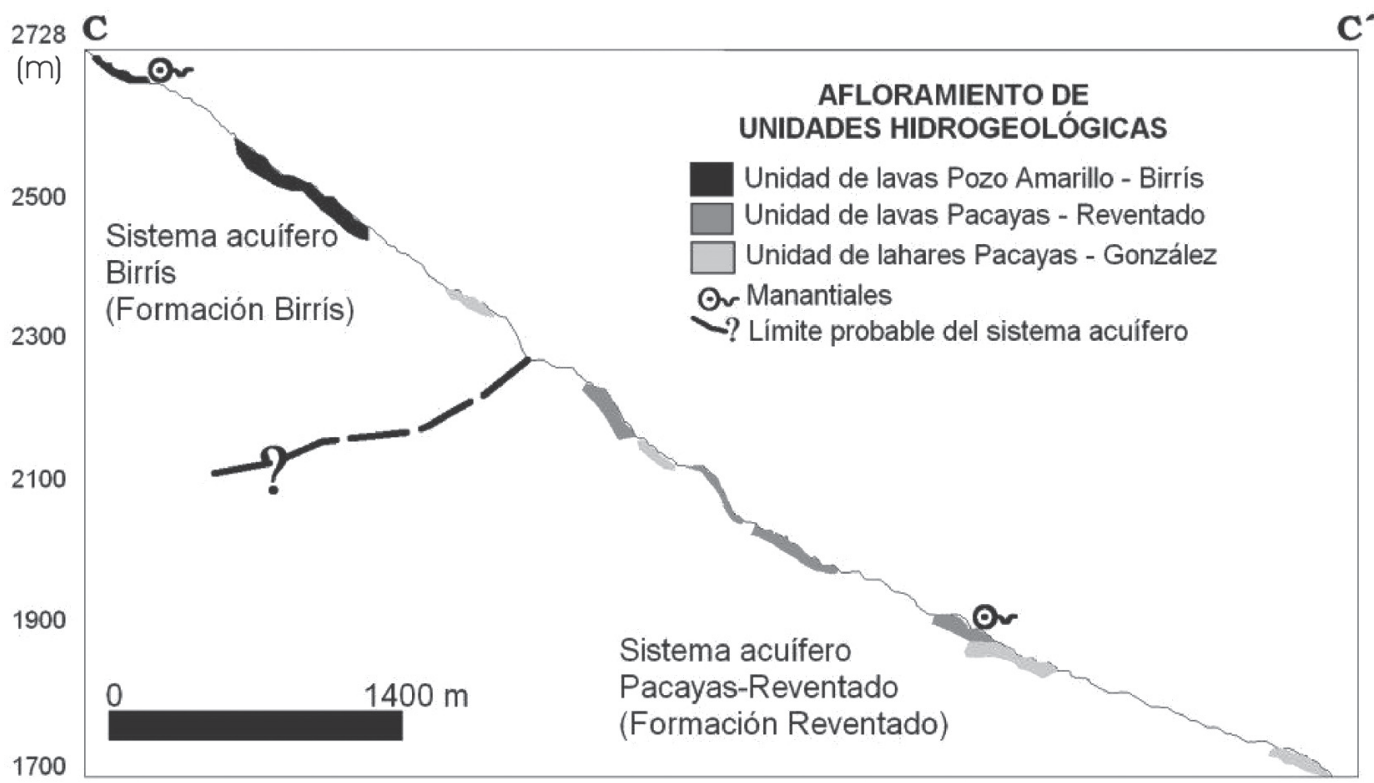

Fig. 6: Sección con unidades hidrogeológicas C-C' de la quebrada Pacayas, mostrado en planta en la figura 7, en el que se muestran las unidades hidrogeológicas y los sistemas acuíferos.

cuenca, los horizontes más superficiales contienen cenizas muy finas y angulosas con lapilli anguloso (Clark, 1993). Los análisis de los suelos derivados de estas cenizas indican que esta unidad presenta texturas muy arenosas con contenidos de finos menores al $40 \%$ del total del material y porosidades entre 60 y $70 \%$, valores altos que pueden ser debidos a la naturaleza de los suelos volcánicos (Mora, 1998). Las velocidades de infiltración obtenidas de las pruebas de campo van desde $9,54 \mathrm{~cm} / \mathrm{d}$ en sectores donde el uso de la tierra es pasto o cultivos y el terreno está muy compactado, hasta $158,4 \mathrm{~cm} / \mathrm{d}$ en las zonas boscosas, de pastos sin ganado y de cultivos, donde la tierra ha sido removida. Sin embargo, el coeficiente de infiltración debe ser aún mas alto en la zona del volcán Irazú, donde las cenizas están más conservadas, existe poca vegetación y no se encuentra una alteración del suelo significativa por actividades antrópicas. Esta unidad, por sus características de permeabilidad actúa como zona de recarga.

\section{Cenizas Pacayas}

Se encuentran en todo el Miembro Superior de la Formación Reventado y en Cervantes. Son cenizas meteorizadas, de espesores métricos variables y con un desarrollo de suelo en sus horizontes más superficiales. Los análisis de suelos indican que esta unidad presenta texturas de arenas limosas y limo elásticos, con un contenido de finos mayor de $50 \%$ del total del material, con porosidades entre 65 y $85 \%$. Aunque estos valores de porosidad son muy altos, Mora (1998) menciona que estas porosidades son normales para suelos derivados de materiales volcánicos. Las velocidades de infiltración varían desde $9,54 \mathrm{~cm} / \mathrm{d}$ en terrenos compactados, de uso agrícola, pastos o urbano, hasta $216 \mathrm{~cm} / \mathrm{d}$ en zonas boscosas, pastos poco intervenidos y cultivos en terrenos removidos. Las propiedades físicas e hidráulicas de estas cenizas pueden variar mucho debido al intenso uso de la tierra para fines agrícolas y para ganado, además del creciente desarrollo urbano en la zona. Esta unidad puede actuar en algunos sectores como zona de recarga.

\section{Lahares indiferenciados río Birrís}

Se encuentra en las formaciones Birrís y el Miembro Superior de Reventado, aflora en varios sectores del río Birrís (Fig. 4) y presenta espesores 
variables de hasta $2 \mathrm{~m}$. En todos los afloramientos observados se reconoce subyaciendo las lavas (Fig. 4). El depósito está conformado por fragmentos volcánicos decimétricos y centimétricos de tamaños no uniformes, con clastos subredondeados y angulares con diferentes grados de meteorización, la matriz es de color café claro a ocre y muestra una textura arenosa muy meteorizada además de un alto contenido de humedad al tacto. Debido a sus características texturales se considera esta unidad como un acuitardo. Considerando tanto su matriz arenosa como las zonas de brotes de agua, se puede decir que esta unidad podría permitir la conexión hidráulica entre estratos, sin embargo se requieren mayores estudios al respecto.

\section{Lahares indiferenciados Pacayas-González}

Se encuentra en la Formación Reventado Miembro Superior y aflora en las quebradas Pacayas (Fig. 6), Central y González. Macroscópicamente muestran una matriz muy arcillosa, texturalmente muy cohesiva y plástica, la cual contiene fragmentos líticos de lavas y escoria, de tamaños centimétricos y decimétricos, subredondeados y redondeados, muy meteorizados. Los depósitos de lahares se encuentran en la mayoría de los afloramientos subyaciendo a los materiales lávicos del Miembro Superior de la Formación Reventado, principalmente en el curso medio de la quebrada Pacayas (Fig. 6) y en la quebrada Central. Por su estado de meteorización y su matriz plástica y arcillosa, estos lahares presentan un comportamiento de acuitardo.

\section{Lavas blocosas de Cervantes}

Estas lavas andesítico basálticas blocosas y escoriáceas están conformadas por bloques escoriáceos y masivos, de tamaños métricos. Las brechas se caracterizan por presentar una textura arenosa con lapilli muy suelto, con espesores de hasta $20 \mathrm{~m}$. No muestran superficialmente un desarrollo significativo de los horizontes del suelo debido a que es un flujo muy reciente (Dóndoli \& Torres, 1954; Krushensky, 1972). Dentro de la cuenca del río Birrís no se han registrado manantiales que broten de estas lavas, además no se observan drenajes superficiales, probablemente debido a la alta permeabilidad que presentan estas lavas. Pruebas de infiltración realizadas en la parte superior de estas lavas muestran valores de infiltración de más de $350 \mathrm{~cm} / \mathrm{d}$. Por ser esta unidad muy blocosa y escoriácea, actúa como una zona de recarga.

\section{Sistemas acuíferos}

En la cuenca del río Birrís se pueden distinguir dos sistemas acuíferos: el Birrís y el Pacayas-Reventado. Debido a que en la cuenca no existe información sobre aguas subterráneas, la definición de los sistemas acuíferos consideró otros criterios como lo son la litología, afloramiento y cambios de concentración de fluoruro en los manantiales.

\section{Litología y afloramiento de manantiales}

En el mapa geológico se observa cómo la distribución de los manantiales se concentra en las formaciones Birrís y Reventado, Miembro Superior (Fig. 3). En la Formación Birrís, el afloramiento de los manantiales se manifiesta en la unidad de lavas Pozo Amarillo-Birrís y en algunos sectores se puede observar cómo esta unidad está subyacida por la unidad de lahares indiferenciados del río Birrís (Fig. 4). De esta manera, la unidad de lahares indiferenciados permite la descarga de agua subterránea de la unidad de lavas Pozo Amarillo-Birrís debido a que presenta una menor permeabilidad por sus características texturales.

En el Miembro Superior de Reventado, el brote de manantiales se observa en la unidad de lavas Pacayas-Reventado (Fig. 5 y 6). Estos afloramientos se observan muy distribuidos en todo el Miembro Superior y en algunos lugares se observa la unidad de lavas sobreyaciendo la unidad de lahares indiferenciados quebradas Pacayas-González o la unidad de lavas meteorizadas Pacayas-Ortiga. Los manantiales del sistema acuífero Pacayas-Reventado se concentran en varios puntos de todo el Miembro Superior, 
sin embargo los afloramientos más importantes corresponden a la zona de manantiales del cañón de la confluencia Central-González (Fig. 5) y la quebrada Pacayas (Fig. 6). En ambos cañones, el brote de agua es a través de la unidad de lavas Pacayas-Reventado. Los demás afloramientos de manantiales se manifiestan en el contacto entre las unidades de lavas Pacayas-Reventado y las lavas meteorizadas Pacayas-Ortiga.

Los manantiales de la quebrada Pacayas se encuentran aproximadamente a $5 \mathrm{~km}$ al sureste del puente hacia San Pablo de Oreamuno. Estos manantiales brotan de la unidad de lavas Pacayas-Reventado, la cual localmente descansa sobre una lava muy meteorizada. Los manantiales de la confluencia Central-González se hallan a $1 \mathrm{~km}$ al este del camino de Pacayas a San Rafael de Irazú y constituyen una zona muy importante de brote de aguas.

\section{Cambios de concentración de fluoruro en los manantiales}

El sistema acuífero Birrís muestra contenidos de fluoruro más altos (Cuadro 1), que el sistema acuífero Pacayas-Reventado (Cuadro 2). Esto se puede observar en los análisis de fluoruro realizados desde inicios de los años noventa hasta el año 2006 (INCIENSA, 2005; AyA, 2006). Esta diferencia de valores coincide además con la distribución geográfica de los manantiales y con su ubicación, considerando el sistema acuífero en el que afloran y la unidad geológica. Las aguas de los manantiales de la cuenca del río Birrís muestran un comportamiento químico muy particular según su distribución geográfica y contenido de fluoruro. Los manantiales de Pozo Amarillo y Birrís, del sistema acuífero Birrís, presentan valores mayores a $1 \mathrm{mg} / \mathrm{L}$ de fluoruro, en tanto aquellos manantiales pertenecientes al sistema acuífero Pacayas-Reventado muestran valores por debajo de $0,8 \mathrm{mg} / \mathrm{L}$. De esta forma, considerando los criterios hidrogeológicos anteriores se pueden definir dos sistemas acuíferos para la cuenca del río Birrís (Fig. 7).

\section{DINÁMICA DE FLUJO DE AGUAS SUBTERRÁNEAS}

La figura 8 muestra el resultado del modelo numérico de flujo utilizando la discretización de la ecuación diferencial de Laplace.

Se puede observar cómo los valores de las equipotenciales son más bajos conforme disminuye la topografía, lo que comprueba una dirección de flujo paralela al terreno y congruente con la dirección de flujo de los drenajes superficiales (Fig. 8).

El modelo muestra cómo la dinámica de flujo de las aguas subterráneas es diferente según el sistema acuífero en el que se encuentren. Utilizando el mapa de sistemas acuíferos (Fig. 7) y el mapa de equipotenciales y dirección de flujo derivados del modelo numérico (Fig. 8) es posible reconocer distintas relaciones hidráulicas.

En el sistema acuífero Birrís se reconoce una relación hidráulica de tipo efluente hacia el río Birrís en toda la zona de manantiales de Pozo Amarillo y los manantiales Birrís (Figs. 7 y 8). La forma de las equipotenciales en estos sectores se

\section{Cuadro 1}

Valores promedio de fluoruro en los manantiales del sistema acuífero Birrís, 1991-2006.

\begin{tabular}{ccc}
\hline Manantial & $\begin{array}{c}\text { Concentración promedio de fluoruro } \\
(\mathrm{mg} / \mathrm{L})\end{array}$ & Año \\
\hline Pozo Amarillo & 1,13 & $1996-2006$ \\
Pozo Amarillo e ICE & 1,23 & $1996-2006$ \\
Naciente Birrís & 1,18 & $1991-2006$ \\
Naciente Brenes & 1,02 & $1991-2006$ \\
\hline
\end{tabular}

Fuente: AyA 2006, INCIENSA, 2005. 
Cuadro 2

Valores promedio de fluoruro en los manantiales del sistema acuífero Pacayas-Reventado, 1991-2006

\begin{tabular}{|c|c|c|c|c|c|c|c|}
\hline Id & Manantial & $\begin{array}{l}\text { Concentración } \\
\text { promedio de fluo- } \\
\text { ruro }(\mathrm{mg} / \mathrm{L})\end{array}$ & Año & Id & Manantial & $\begin{array}{l}\text { Concentración } \\
\text { promedio de } \\
\text { fluoruro }(\mathrm{mg} / \mathrm{L})\end{array}$ & Año \\
\hline 1 & Buenavista & 0,26 & $96-06$ & 9 & Marcos Pelé & 0,10 & $96-06$ \\
\hline 2 & $\begin{array}{c}\text { Aníbal } \\
\text { Barquero }\end{array}$ & 0,12 & $96-06$ & 10 & El Descanso & 0,53 & $96-06$ \\
\hline 3 & Padre Coto & 0,10 & $96-06$ & 11 & Noré Gómez & 0,10 & $96-06$ \\
\hline 4 & La Vivienda & 0,30 & $96-06$ & 12 & Las Aguas & 0,11 & $96-06$ \\
\hline 5 & $\begin{array}{c}\text { Minor } \\
\text { Fernández }\end{array}$ & 0,46 & $96-06$ & 13 & $\begin{array}{l}\text { Matías } \\
\text { Solano }\end{array}$ & 0,53 & $96-06$ \\
\hline 6 & $\begin{array}{c}\text { Martín } \\
\text { Montero }\end{array}$ & 0,55 & $96-06$ & 14 & $\begin{array}{c}\text { Rafael } \\
\text { Sandoval }\end{array}$ & 0,26 & $96-06$ \\
\hline 7 & $\begin{array}{l}\text { Vicente } \\
\text { Serrano }\end{array}$ & 0,20 & $96-06$ & 15 & $\begin{array}{c}\text { María } \\
\text { Cristina }\end{array}$ & 0,46 & $96-06$ \\
\hline 8 & Lalo Leandro & 0,64 & $96-06$ & 16 & La Tica & 0,20 & $96-06$ \\
\hline
\end{tabular}

Fuente: AyA 2006, INCIENSA, 2005.

comprueba por el afloramiento de la unidad de lavas Pozo Amarillo-Birrís y por el brote de manantiales. Los aforos diferenciales realizados en distintos puntos del río Birrís indican un aumento de caudal conforme el río avanza. En los manantiales Pozo Amarillo el caudal del río Birrís fue de 3,62 L/s, en el sector de La Floresta, $1 \mathrm{~km}$ antes de los manantiales Birrís el caudal aumentó a 11,08 L/s, luego del paso por las manantiales Birrís, el caudal del río aumentó a 50,01 L/s, lo cual indica el importante aporte de agua que brindan estos manantiales y lo cual se observa a partir de la dirección de flujo en el mapa de equipotenciales (Fig. 8).

En el sector este de la cuenca, las líneas equipotenciales no reflejan concavidad alguna, excepto en la quebrada González (Figs. 7 y 8) donde hay un comportamiento influente el cual se refleja en su forma. Esta característica se corrobora con el poco caudal que muestra esta quebrada durante todo el año. Con excepción del río Birrís, todos los drenajes en este sistema acuífero mantienen muy poco caudal durante la época seca, lo que puede indicar que las descargas del sistema acuífero Birrís se dan principalmente a ese río (Fig. 8).

En el sistema acuífero Pacayas-Reventado, el modelo de flujo muestra un comportamiento de las equipotenciales distinto al sistema acuífero Birrís. Se muestran dos zonas de conexión hidráulica efluente, los manantiales de la confluencia Central-González y los manantiales de la quebrada Pacayas (Figs. 7 y 8). En la confluencia Central-González se reconoce una importante zona de manantiales brotando de la unidad de lavas Pacayas-Reventado, la cual coincide con la forma de las equipotenciales (Figs. 7 y 8). El aumento del caudal de esta quebrada explica el motivo de que antes de la catarata donde está el sector de manantiales, el caudal de la confluencia fue de $2,64 \mathrm{~L} / \mathrm{s}$ y luego de ese punto el caudal aumenta a más de $60 \mathrm{~L} / \mathrm{s}$, según la información que tienen las asociaciones de acueductos rurales.

En la quebrada Pacayas (Figs. 7 y 8), el comportamiento efluente también es coincidente con los manantiales aflorantes y con el aumento de caudal de esta quebrada en el sistema acuífero Pacayas-Reventado. La medición del caudal realizada en esta quebrada $5 \mathrm{~km}$ antes de la zona de manantiales fue de $4,94 \mathrm{~L} / \mathrm{s}$ y una vez que pasa por este punto el caudal aumenta hasta $80,8 \mathrm{~L} / \mathrm{s}$. Este comportamiento se refleja en esta quebrada y contrasta con la influencia que existe en los otros drenajes que muestran caudales muy bajos durante toda la época seca. 


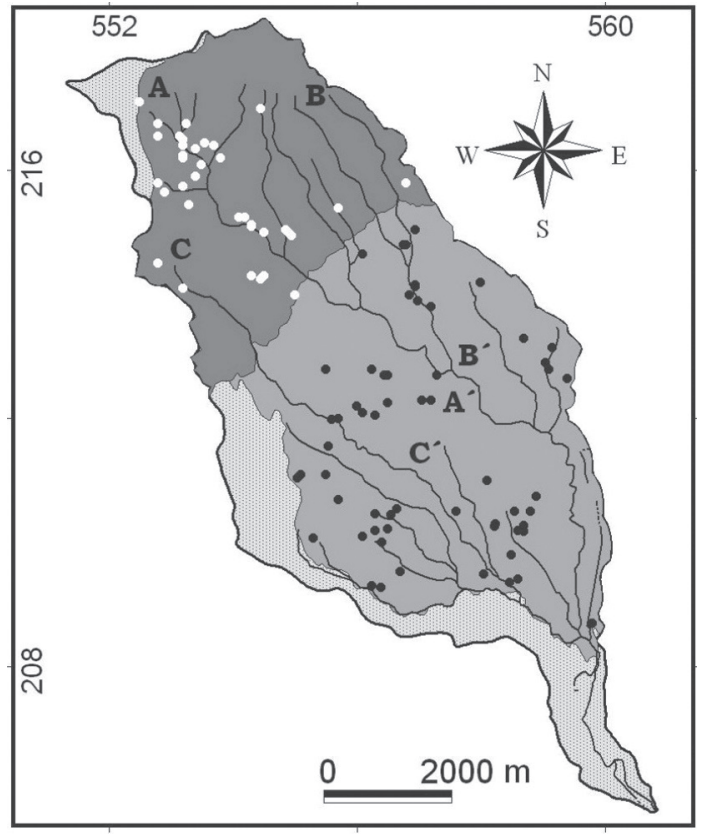

Manantiales Birrís Afloramientos Manantiales Pacayas de unidades $\sim$ Ríos y quebradas hidrogeológicas

\begin{tabular}{ll} 
Sistemas acuíferos & A-A' \\
$\square$ Birrís & B-B' \\
\hline Pacayas-Reventado & C-C' \\
Zona no acuífera &
\end{tabular}

Fig. 7: Mapa de sistemas acuíferos definidos dentro de la cuenca del río Birrís. Se incluyen los manantiales, los drenajes superficiales y las líneas de afloramientos de unidades hidrogeológicas a lo largo de los drenajes principales.

\section{Comparación del modelo numérico y otro método aplicado en hidrogeología}

Además de la utilización del modelo numérico en régimen permanente, se aplicó a los datos de manantiales y drenajes superficiales un método de interpolación espacial estadístico conocido como Kriging (Fig. 9). Este método ha sido muy usado en hidrogeología para construir las equipotenciales y para comparar las curvas generadas a partir del método numérico (Marsily, 1986; Anderson \& Woessner, 1992; Kitanidis, 1993; Knapp, 1999; Kumar \& Remadevi, 2006).
El método de Kriging asume que la variable de estudio es una función aleatoria cuya correlación espacial está definida por un variograma, el cual es un gráfico que muestra la medida del cambio en la variable conforme aumenta la distancia (Journel \& Huijbregts, 1978; Isaaks \& Srivastava, 1989; Anderson \& Woessner, 1992). Una correlación alta entre puntos medidos es esperada para distancias cortas, siendo baja en caso contrario.

El fundamento de este método ha sido bien documentado (Journel \& Huijbregts, 1978; Isaaks \& Srivastava, 1989) y su aplicación a la hidrogeología es de gran utilidad, debido a que permite estimar valores desconocidos a través de un grupo de puntos conocidos, en este
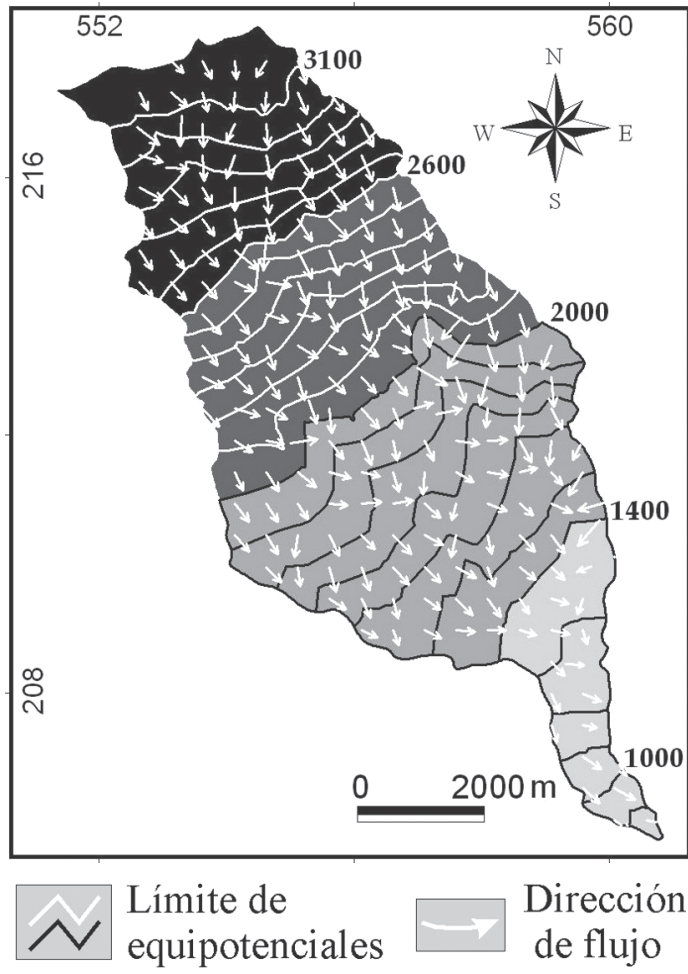

Equipotenciales (m.s.n.m)

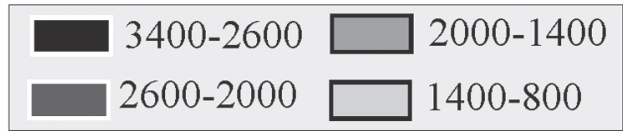

Fig. 8: Mapa de equipotenciales y dirección de flujo construidas mediante un modelo hidrogeológico numérico. 


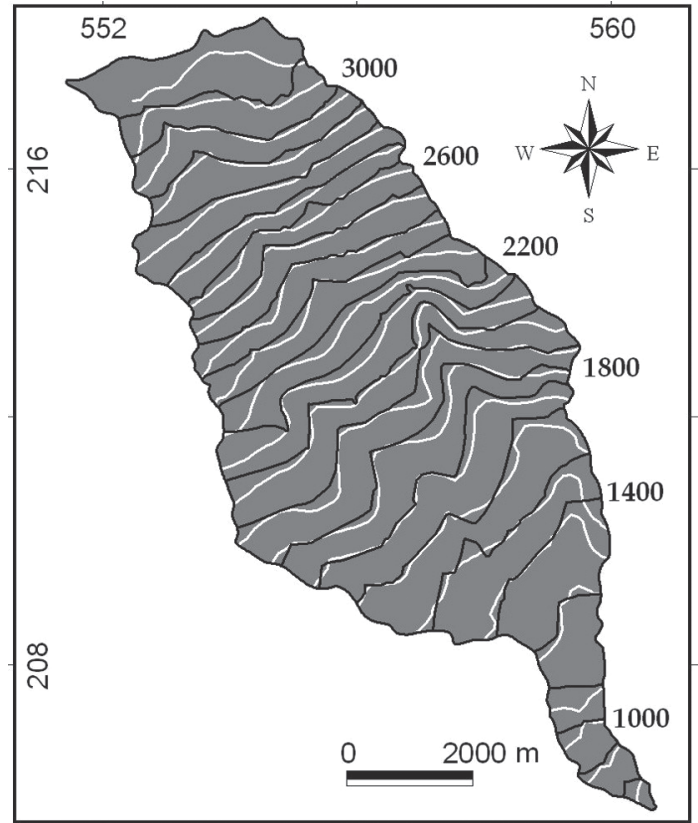

Equipotenciales modelo numérico Equipotenciales modelo estadístico

Fig. 9: Mapa de equipotenciales donde se compara el modelo hidrogeológico numérico y el modelo estadístico. Nótese la similitud de ambos modelos.

caso a partir de los manantiales ubicados en una zona específica (Marsily, 1986; Kitanidis, 1993; Knapp, 1999; Kumar \& Remadevi, 2006).

En la figura 9 se muestran las equipotenciales construidas por medio del modelo numérico de flujo en régimen permanente y las generadas por medio del método de interpolación estadístico de Kriging. Se puede observar cómo los contornos generados por ambos métodos son muy semejantes. En ambos las zonas de efluencia son bien indicadas, lo que comprueba la distribución de los manantiales y el aumento de caudal de los drenajes superficiales en esos puntos.

La ventaja del modelo numérico sobre el estadístico radica en que este está fundamentado en la ecuación de Laplace, que constituye una de las ecuaciones de mayor uso en hidrogeología, considera condiciones de frontera y calcula el valor de carga hidráulica celda por celda (PolubarinovaKochina, 1962; Bear, 1972, 1979; Marsily, 1986;
Anderson \& Woessner, 1992). Conforme se aumenten los datos del modelo conceptual, como los valores de recarga y descarga, estos pueden agregarse a la ecuación de Laplace y aumentar aún más la precisión de las equipotenciales, lo que no se puede realizar con el método de Kriging, pues solo considera la distribución espacial de los valores de carga hidráulica.

\section{CONCLUSIONES Y RECOMENDACIONES}

En el área de estudio se definieron dos sistemas acuíferos: Birrís y Pacayas-Reventado, pertenecientes a las formaciones Birrís y el Miembro Superior de Reventado, respectivamente. Estos están conformados por materiales volcánicos, los cuales constituyen unidades hidrogeológicas como lavas, lahares y cenizas. Cada una de estas unidades muestra un comportamiento diferente al paso del agua, lo que ha permitido describirlas como acuíferos, acuitardos o zonas de recarga. Los sistemas acuíferos se diferencian por la distribución de los manantiales según la litología y por su concentración de fluoruro.

La aplicación de un modelo hidrogeológico numérico en régimen permanente utilizando Microsoft Excel $\mathbb{C}$ es una herramienta importante para caracterizar la dinámica de flujo de las aguas subterráneas en zonas donde no existen pozos y la información hidrogeológica sea muy limitada. Sin embargo, deben existir datos de elevación de niveles equipotenciales. De esta manera se pudieron crear las líneas equipotenciales y posteriormente determinar la dirección de flujo y con base en esta información, establecer el comportamiento hidráulico de las aguas subterráneas y su relación con los drenajes superficiales. A partir de estos datos, se establecieron las zonas de efluencia e influencia, las cuales son congruentes con las observaciones de las variaciones de los caudales de los aforos diferenciales realizados en campo. Este modelo puede verse enriquecido conforme se avance en el conocimiento teórico del modelo conceptual de manera que sea posible integrarle recargas, descargas, transmisividad y almacenamiento, entre otros.

El método estadístico de Kriging es una herramienta útil para generar las equipotenciales, sin 
embargo su aplicación es muy limitada con respecto al modelo numérico. Conforme se aumente el conocimiento del modelo hidrogeológico conceptual, la información se puede agregar a cada celda del modelo numérico llegando incluso a convertir la ecuación de flujo permanente a flujo transitorio. De esta forma las ventajas del modelo numérico son mucho mayores que las del modelo estadístico.

El principal aporte de esta investigación fue la definición de un modelo numérico hidrogeológico que mejora el modelo conceptual en el cual se describen las unidades y los sistemas acuíferos en la cuenca del río Birrís. Sin embargo, hay que mencionar que la información hidrogeológica de esta cuenca y de su comportamiento hidráulico es aún muy poca con respecto a otras zonas del país, por lo que es recomendable poder realizar mayores investigaciones en esta zona.

\section{AGRADECIMIENTOS}

A mis compañeras Laura Castro y Laura Gil por el apoyo en este trabajo, al M.Sc. Gunther Schosinsky por sus consejos y observaciones para desarrollar el modelo y por la revisión del artículo, al Ph.D. Jaime Gárfias por las sugerencias en el inicio de la construcción del modelo numérico, a los geólogos M.Sc. Luis Obando, M.Sc. Mario Arias y M.Sc. Giovanni Peraldo por sus sugerencias y comentarios para este trabajo, a las asociaciones administradoras de acueductos rurales de Santa Rosa, San Pablo de Oreamuno y San Rafael de Irazú y la Municipalidad de Alvarado, al Laboratorio de Geotecnia de la Escuela de Geología por la recolección y procesamiento de los análisis de suelos, a INCIENSA y AyA por los datos de fluoruro y al proyecto CARA por el financiamiento parcial de esta investigación.

\section{REFERENCIAS}

ALLÈGRE, C. \& CONDOMINES, M., 1976: Fine Chronology of Volcanic Processes using 238U - 230Th Systematics.- Earth Plan. Sci. Let. 28, 395-406.
ALVARADO, G., 1993: Volcanology and Petrology of Irazú Volcano, Costa Rica.- 261 págs, Univ. Kiel, Alemania, [Tesis Ph.D].

ALVARADO, G.E., CARR, M.J., TURRIN, B.D., SWISHER III, C.C., SCHMINCKE, H.-U. \& HUDNUT, K.W., 2006: Recent volcanic history of Irazú volcano, Costa Rica: Alternation and mixing of two magma batches, and pervasive mixing. - En: Rose, W.I., BLUTH, G.J.S., CARR, M.J., EWERT, J.W., PATINO, L.C. \& VALLANCE, J.W. (Eds.): Volcanic hazards in Central America. Geol. Soc. Amer. Sp. Paper, 412: 259-276.

ANDERSON, M. \& WOESSNER, W., 1992: Applied Groundwater Modeling: Simulation of Flow and Advective Transport.- 381 págs. Academic Press, Nueva York.

ASTM., 2004: Standard Guide for Establishing Nomenclature of Ground-Water Aquifers.17 págs, ASTM International.

AyA, 2006: Informe de Calidad de Agua Potable.10 págs. Lab. Nac. Aguas, Costa Rica.

BEAR, J., 1972: Dynamics of Fluids in Porous Media.- 784 págs. Dover, Nueva York.

BEAR, J., 1979: Hydraulics of Groundwater.-569 págs, Dover, Nueva York.

CLARK, S., 1993: The Recent Eruptive History of Irazú Volcano, Costa Rica: A Study of Tephra Deposits of the Last 2500 years with Geochemical and Isotopic Analysis of the 1963-65 Eruption.-186 págs. Univ. Iowa, Estados Unidos, [Tesis M.Sc.].

CUSTODIO, E. \& LLAMAS, M.R., 2001: Hidrología Subterránea.- 2350 págs, Omega, Barcelona.

DAVIS, S.N. \& DE WIEST, R.J.M., 1966: Hydrogeology.- 563 págs, Wiley, Nueva York. 
DÓNDOLI, C. \& TORRES, J., 1954: Estudio Geoagronómico de la Región Oriental de la Meseta Central.-180 págs, Ministerio de Agricultura e Industrias, San José, Costa Rica.

INCIENSA, 2005: Concentración de Flúor en el Agua de los Cantones Alvarado y Oreamuno.- INCIENSA, Tres Ríos [Inf. interno].

ISAAKS, E. \& SRIVASTAVA, R., 1989: An Introduction to Applied Geostatistics.- 560 págs, Oxford, Nueva York.

JOURNEL, A. \& HUIJBREGTS, C., 1978: Mining Geostatistics.- 600 págs. Academic Press, Londres.

KITANIDIS, P., 1993: Geostatistics. - En: MAIDMENT, D. (ed): Handbook of Hydrology.- 2947 págs, McGraw Hill, Nueva York.

KNAPP, K., 1999: The Interpolation of Hydraulic Head Contours.- Department of Mathematics \& Computer Science, Ursinus Collage, [Informe interno].

KRUSHENSKY, R., 1972: Geology of the Istarú Quadrangle, Costa Rica.- US Geol. Surv. Bull. 1358: 1-83.
KUMAR, V. \& REMADEVI., 2006: Kriging of Groundwater Levels - A case study.- J. Spat. Hydrol. 6(1).

MANGA, M., 2001: Using Springs to Study Groundwater Flow and Active Geologic Processes.- An. Rev. Earth Planet. Sc. 29: 201-228.

MARSILY,G., 1986:QuantitativeHydrogeology.440 págs. Academic Press, Nueva York.

MINAE, 2006: Reporte Técnico de Concesiones Detalladas para los Cantones de Oreamuno, Alvarado y Jiménez.- 93 págs. San José, [Inf. interno].

MORA, R., 1998: Propiedades físicas, hidráulicas y mecánicas de suelos de origen volcánico, en sitios seleccionados del Valle Central, Costa Rica.- Rev. Geol. Amér. Central, 21: 81-98.

POLUBARINOVA-KOCHINA, P.YA., 1962: Theory of Ground Water Movement.- 613 págs. Traducción del ruso por J.M. Roger de Wiest, Princeton, Nueva Jersey.

THORPE, R.S. \& BROWN, G.C., 1985: The field description of igneous rocks.- 154 págs. Geol. Soc. London. 
